\title{
Multiprecision multiplication on AVR revisited
}

\author{
Michael Hutter · Peter Schwabe
}

July 15, 2014

\begin{abstract}
This paper presents new speed records for multiprecision multiplication on the AVR ATmega family of 8bit microcontrollers. For example, our software takes only 1998 cycles for the multiplication of two 160-bit integers; this is more than $14 \%$ faster than previous results. For 256bit inputs, our software is not only the first to break through the 6000-cycle barrier; with only 4797 cycles it also breaks through the 5000-cycle barrier and is more than $20 \%$ faster than previous work. We achieve these speed records by carefully optimizing the Karatsuba multiplication technique for AVR ATmega. One might expect that subquadratic-complexity Karatsuba multiplication is only faster than algorithms with quadratic complexity for large inputs. This paper shows that it is in fact faster than fully unrolled product-scanning multiplication already for surprisingly small inputs, starting at 48 bits. Our results thus make Karatsuba multiplication the method of choice for high-performance implementations of elliptic-curve cryptography on AVR ATmega microcontrollers.
\end{abstract}

Keywords Karatsuba multiplication - microcontroller · ATmega.

This work was supported by the Austrian Science Fund (FWF) under the grant number TRP251-N23 and by the Netherlands Organisation for Scientific Research (NWO) through Veni 2013 project 13114. Permanent ID of this document: $102 \mathrm{fe} 77 \mathrm{c} 6 \mathrm{~d} 1003 \mathrm{e} 5694 \mathrm{ac04543a52410.}$

Michael Hutter

Graz University of Technology

Institute for Applied Information Processing and Communications

Inffeldgasse 16a, A-8010, Graz, Austria

E-mail: michael.hutter@iaik.tugraz.at

Peter Schwabe

Radboud University Nijmegen

Digital Security Group

PO Box 9010, 6500GL Nijmegen, The Netherlands

E-mail: peter@cryptojedi.org

\section{Introduction}

How much effort is it to multiply two integers? Over the last 6 decades, many researchers have attempted to answer this question. One main line of research is concerned with the asymptotic complexity of integer multiplication. In the 1950s, Kolmogorov conjectured that the complexity for the multiplication of two $n$-digit integers is $\Theta\left(n^{2}\right)$. This conjecture was proven wrong by one of his students, Karatsuba, in 1960 who presented a multiplication algorithm with asymptotic complexity $\Theta\left(n^{\log _{2} 3}\right)$ This ground-breaking result was published in 1962 [13]. See [14, Section 6] for a history of this publication. In 1963, Toom lowered the complexity further to $n 2^{\Theta\left(\sqrt{\log _{2} n}\right)}$ [26] and the FFT-based method by Schönhage and Strassen achieves asymptotic complexity $\Theta(n \log n \log \log n)$ [20]. The latest result in this line of research is Fürer's algorithm with asymptotic complexity $\Theta\left(n \log n 2^{\log ^{*} n}\right)[4]$.

Another main line of research is concerned with the number of clock cycles required for multiplication of two integers of a particular fixed size on a particular micro-architecture. This paper presents new results from this second line of research. Specifically, we present new multiplication speed records for multiplication of integers between 64 and 256 bits on the AVR ATmega family of microcontrollers. We expect that the techniques described in this paper can be extended to larger inputs. However, in this paper we are mainly interested in input sizes in the range between 160 and 256 bits that we typically encounter in elliptic-curve cryptography on embedded processors. The software presented in this paper takes time independent of the inputs and is thus suitable for use in timing-attack-protected implementations of cryptographic primitives.

All previous speed records for multiplication of integers of up to 256 bits on AVR are achieved by algorithms with quadratic complexity. We obtained our speed records by op- 
timizing the Karatsuba multiplication algorithm for the AVR ATmega architecture. We do not claim novelty for any particular technique we used. What is new is the combination of techniques and careful hand-optimization of multiprecision multiplication for AVR ATmega microcontrollers.

Notes on naming. While working on this paper we observed that the term "schoolbook multiplication" has a different meaning for different people and in different contexts. Sometimes, it only refers to operand-scanning multiplication. Other techniques with quadratic complexity, such as product-scanning multiplication, hybrid multiplication [8], or operandcaching multiplication [11] are not considered to be "schoolbook". For an example of this naming convention, see [11, Section 3]. However, when distinguishing multiplication algorithms with different asymptotic complexities, "schoolbook multiplication" is often used to refer to any quadraticcomplexity algorithm. See, for example, [8, Section 3]. To avoid confusion we avoid the term "schoolbook multiplication" throughout this paper.

It is common to refer to the product-scanning technique as "Comba multiplication", and to give credit to a 1990 paper by Comba [3]. See, for example, [21,5,11]. However, the technique has earlier been described (without a claim of novelty) by Barrett in [1, Diagram three]. The method has in fact already been described by Leonardo Pisano (Fibonacci) in his work "Liber Abaci" from 1202; see [24, Chapter 2]. Swetz in [25, Chapter 4] states that the "cross method of multiplication" can be traced back to the Lìlâvatī by Bhāskara from 1150, but we were not able to confirm this in the English translation by Patwardhan, Naimpally, and Singh [19]. We will use the term "product-scanning multiplication".

Related work. Many results exist on fast multiprecision multiplication on embedded processors, often in the context of modular arithmetic and elliptic-curve cryptography. Some papers also consider the Karatsuba technique for multiplication on embedded processors. For example, Großschädl, Avanzi, Savas, and Tillich use the Karatsuba technique for fast and energy-efficient multiplication of 512-bit and larger integers on StrongARM [7]; Gouvêa and López use Karatsuba for 256-bit multiplication on the MSP430 [5]; Gouvêa, Oliveira, and López use it for fast 256-bit multiplication on the MSP430X [6].

On AVR ATmega microcontrollers the state of the art in multiplication of integers of up to 256 bits has consistently been held by algorithms with quadratic complexity. Until 2004 the fastest known algorithm was product-scanning multiplication. For inputs of size larger than 96 bits this changed with the introduction of hybrid multiplication by Gura, Patel, Wander, Eberle and Chang Shantz in [8]. This algorithm was later improved by Scott and Szczechowiak in [21]. The next milestone in optimizing multiprecision multiplication on AVR was the best paper of CHES 2011 by Hutter and Wenger that introduced operand caching multiplication [11]. The results of that paper were slightly improved in two followup papers by Seo and Kim, which introduced and optimized consecutive operand caching [22,23]. These papers mark the current state of the art in multiprecision multiplication on AVR ATmega microcontrollers. Note that the use of hybrid multiplication is covered by a patent [9]; a patent for operand-caching multiplication is pending [12].

We are aware of only two papers considering the Karatsuba technique for multiplication of big integers on AVR ATmega. Both papers conclude that Karatsuba multiplication is slower than quadratic-complexity multiplication algorithms for input sizes commonly used in elliptic-curve cryptography. In [17], Liu, Großschädl, and Kizhvatov consider different approaches for implementing Montgomery multiplication including "Hybrid Karatsuba-Comba Multiplication (HKCM)". They conclude in Section III.B that

\section{"The HKCM method is faster than HSOS (and also HFIPS) for operands exceeding 512 bits in length, but slower in the case of 256-bit operands. [...] For 512-bit operands, the HKCM method achieves es- sentially the same performance as HSOS, and both are roughly $7 \%$ faster than HFIPS."}

Both "Hybrid Separated Operand Scanning" (SOS) and "Hybrid Finely Integrated Product Scanning" (HFIPS) are algorithms with quadratic complexity.

In [10], we used Karatsuba for multiplication of 256-bit integers; however, with 6686 cycles, that approach turned out to be considerably slower than state-of-the-art operandcaching and consecutive operand-caching multiplication.

Availability of Software. We will place the software described in this paper into the public domain and make it available online. We will not apply for any patents for the techniques described in this paper.

Organization of the paper. Section 2 briefly reviews the specifics of the AVR ATmega family of microcontrollers. Section 3 first considers efficient approaches for small multiprecision multiplication, then discusses different approaches for implementing Karatsuba multiplication on AVR ATmega, and finally derives a lower bound on the number of cycles purely from arithmetic instructions. Section 4 describes how we minimize the number of loads and stores in Karatsuba multiplication for different input sizes to translate the lower computational complexity to lower cycle counts. In Section 5 we present detailed performance results of our software and compare with the best results from the literature. We conclude the paper and give ideas for future work in Section 6. 


\section{The AVR ATmega architecture}

This paper optimizes Karatsuba multiplication for the AVR ATmega family of 8-bit microcontrollers. Many of the techniques we describe apply in a similar way on other architectures, but the concrete application of these techniques and the cost analysis is specific to the 8-bit AVR ATmega architecture. This section briefly reviews the specifics of this architecture that are relevant to the remainder of the paper.

Register set. The AVR has 32 registers labeled R0,.., R31. The register pair (R26,R27) is aliased as X, the register pair (R28,R29) is aliased as Y, and the the register pair (R30,R31) is aliased as $\mathrm{Z}$. These three register pairs are the only ones that can hold the address argument of a load or store instruction. The register pair (R0,R1) is special because it holds the output of a multiplication instruction (see below).

Memory access. All load and store instructions on the ATmega take 2 cycles. The LD load instruction and the ST store instruction access memory at the address specified in their argument (either X, Y, or Z). They can post-increment or predecrement their 2-register argument for free. The LDD load instruction takes a constant offset to the address register as second argument; so does the STD store instruction.

The standard way to use the stack is to use the instructions PUSH and POP. However, it is also possible to use two IN instructions to copy the stack pointer into one of the address register pairs $\mathrm{X}, \mathrm{Y}$, or $\mathrm{Z}$ and then operate on the stack with LD/LDD and ST/STD instructions. Writing back the stack pointer takes two OUT instructions

Arithmetic instructions. Our software makes use of only relatively few arithmetic instructions. Most important is the MUL instruction, which multiplies the 8-bit unsigned integers in its two register arguments; the 16-bit result is written to the register pair (R0,R1). The MUL instruction takes 2 cycles and it overwrites the carry flag. Addition (ADD/ADC), subtraction (SUB,SBC), and exclusive or (EOR) are two-operand instructions; one of their inputs is overwritten by the output. For subtraction it is always the minuend that is overwritten. Another helpful instruction is SBCI which performs subtraction with borrow of an immediate value from a register. There is no equivalent "ADCI" instruction to perform addition with carry of an immediate value. The CLR instruction sets a register to zero; the MOV instruction copies the value in one register to another register. The MOVW instruction copies a register pair to another register pair. Note that two adjacent registers are a register pair only if the lower register is "even" (i.e., R0, R2, ...). It is worth noting that MOVW, like all other arithmetic instructions except MUL, takes only one cycle.

Aside from the typical flags (like carry, zero, etc.), the AVR also features a T flag, which can be used to remember a single bit. The BST instruction stores one bit of a given register to the $\mathrm{T}$ flag, the BLD instruction loads from the $\mathrm{T}$ flag into one bit of a given register. It is possible to perform conditional branches depending on the value of the T flag.

C function-call ABI. The avr-gcc function-call ABI specifies that the first three 16-bit arguments (e.g., pointers) are passed in register pairs (R24,R25), (R22,R23), and (R20,R21). It furthermore specifies that registers R2-R17, R28, and R29 are caller registers, and that register $\mathrm{R} 1$ has to be set to zero before returning from a function. Our software follows these conventions to make it directly usable from $\mathrm{C}$ code, but as previous paper we do not include function-call-ABI related overhead in our cycle counts. See Section 5.

\section{Arithmetic considerations}

In this section we consider the pure arithmetic cost, i.e., ignoring costs for loads and stores, of Karatsuba multiplication on AVR ATmega. We start with fixing the representation of big integers and reviewing the arithmetic cost of small multiprecision multiplications to establish a baseline.

Representation of big integers. Throughout this paper we will represent big integers in unsigned radix- $2^{8}$ representation, i.e., an $8 m$-bit integer $A$ is represented in $m$ bytes $\left(a_{0}, \ldots, a_{m-1}\right)$ with $A=\sum_{i=0}^{m-1} a_{i} 2^{8 i}$ and $a_{i} \in\{0, \ldots, 255\}$. This big-integer representation is standard for AVR throughout the literature. We do not expect any benefits from using a signed representation or a "carry-safe" representation, which leaves some bits on the top of each limb free to accumulate carries.

Small multiprecision multiplications. Karatsuba multiplication, like hybrid multiplication, constructs full-size multiprecision multiplication from blocks of smaller multiplications. The block sizes that are most relevant for this paper are $24 \times 24$ bits, $32 \times 32$ bits, $40 \times 40$ bits, and $48 \times 48$ bits. One obvious way to handle those "small multiprecision" multiplications is to use operand scanning or product scanning. However, this is not optimal as demonstrated in the context of inner-loop optimization for the hybrid multiplication by Lederer, Mader, Koschuch, Großschädl, Szekely, and Tillich in [15], by Liu, Großschädl, and Kizhvatov in [17], and most recently by Liu and Großschädl in [16].

For $32 \times 32$-bit multiplication we adapted the technique described in [16, Section 3.1] for multiplication (the original algorithm performs multiply-accumulate). Inspired by this technique we wrote similar routines for $24 \times 24$-bit for $40 \times 40$-bit, and for $48 \times 48$-bit multiplication. Table 1 lists instruction and cycle counts for those small multiplications; the corresponding code listings are in Appendix B. Note that our routines are slightly different from the one by Liu in the sense that they can be understood as tweaked operandscanning. We assume that inputs are already loaded to registers and that outputs are also kept in registers. The cost 
Table 1 Instruction and cycle counts for small multiprecision multiplications without loads and stores. Corresponding counts of fully unrolled product-scanning multiplication are listed in parentheses.

\begin{tabular}{|c|r|r|r|r|r|}
\hline Input size & MUL & ADD/ADC & MOV (W) & CLR & cycles \\
\hline $24 \times 24$ bit & 9 & 17 & 4 & 3 & 42 \\
& $(9)$ & $(22)$ & $(1)$ & $(5)$ & $(46)$ \\
\hline $32 \times 32$ bit & 16 & 35 & 6 & 3 & 76 \\
& $(16)$ & $(43)$ & $(1)$ & $(7)$ & $(83)$ \\
\hline $40 \times 40$ bit & 25 & 58 & 8 & 2 & 118 \\
& $(25)$ & $(70)$ & $(1)$ & $(9)$ & $(130)$ \\
\hline $48 \times 48$ bit & 36 & 83 & 15 & 2 & 172 \\
& $(36)$ & $(103)$ & $(1)$ & $(11)$ & $(187)$ \\
\hline
\end{tabular}

for loads and stores depends on the context in which these multiplication blocks are used.

We do not claim speed records for these small multiplications, although we are not aware of any faster results. We would expect that there exist thoroughly optimized routines for these input sizes that are in the range of standard C data types. We were surprised to find the currently fastest approach somewhat "hidden" as an inner-loop optimization of big-integer hybrid multiplication in a paper on Montgomery modular multiplication.

Note that the optimized small multiprecision multiplications need slightly more live registers than fully unrolled product-scanning multiplication. Whether they are better than product scanning or not depends on the context, i.e., the amount of registers that are available without spilling.

Additive vs. subtractive Karatsuba. From now on we are considering $n \times n$-byte multiplication, where $n$ is even and $k=n / 2$. The typical way to describe Karatsuba multiplication of an $n$-byte integer $A \hat{=}\left(a_{0}, \ldots, a_{n-1}\right)$ and and $n$-byte integer $B \hat{=}\left(b_{0}, \ldots, b_{n-1}\right)$ is the following:

- Write $A=A_{\ell}+2^{8 k} A_{h}$ and $B=B_{\ell}+2^{8 k} B_{h}$ for $k$-byte integers $A_{\ell}, A_{h}, B_{\ell}$, and $B_{h}$;

- compute $L=A_{\ell} B_{\ell} \hat{=}\left(\ell_{0}, \ldots, \ell_{n-1}\right)$;

- compute $H=A_{h} B_{h} \hat{=}\left(h_{0}, \ldots, h_{n-1}\right)$;

- compute $M=\left(A_{\ell}+A_{h}\right)\left(B_{\ell}+B_{h}\right) \hat{=}\left(m_{0}, \ldots, m_{n}\right)$; and

- obtain the result as $A B=L+2^{8 k}(M-L-H)+2^{8 n} H$.

We will refer to this approach as additive Karatsuba. The problem with this approach is that the additions of two $k$ byte numbers $A_{\ell}+A_{h}$ and $B_{\ell}+B_{h}$ produce carry bits. An efficient way to handle multiplications by such a carry bit during the computation of $M$ is to perform a subtractionwith-carry from a zero register to produce a register that is either $0 x f f$ (if the carry is one) or zero and then compute multiplication through an and instruction with this register. Subsequent accumulation of the one-byte result of such a multiplication costs only two addition instructions (one ADD and one ADC) instead of three instructions for two-byte results.
The problem with this approach is twofold: First the multiplications by carry bits still contribute a significant overhead. Second the tweak to use and instructions only works for a single carry bit. Recursive application of Karatsuba's technique yields multiple carry bits which have to be handled by full multiplication and accumulation. It turns out to be more efficient to use subtractive Karatsuba:

- Write $A=A_{\ell}+2^{8 k} A_{h}$ and $B=B_{\ell}+2{ }^{8 k} B_{h}$ for $k$-byte integers $A_{\ell}, A_{h}, B_{\ell}$, and $B_{h}$;

- compute $L=A_{\ell} B_{\ell} \hat{=}\left(\ell_{0}, \ldots, \ell_{n-1}\right)$;

- compute $H=A_{h} B_{h} \hat{=}\left(h_{0}, \ldots, h_{n-1}\right)$;

- compute $M=\left|A_{\ell}-A_{h}\right| \cdot\left|B_{\ell}-B_{h}\right| \hat{=}\left(m_{0}, \ldots, m_{n-1}\right)$;

- set $t=0$, if $M=\left(A_{\ell}-A_{h}\right) \cdot\left(B_{\ell}-B_{h}\right) ; t=1$ otherwise;

- compute $\hat{M}=(-1)^{t} M=\left(\hat{m}_{0}, \ldots, \hat{m}_{n-1}\right)$; and

- obtain the result as $A B=L+2^{8 k}(L+H-\hat{M})+2^{8 n} H$.

This variant of Karatsuba avoids the carry bits in the computation of $M$ but instead needs to compute two absolute differences $\left|A_{\ell}-A_{h}\right|$ and $\left|B_{\ell}-B_{h}\right|$ and one conditional negation of $M$. This has to be done in constant time to make the multiplication routine suitable for timing-attack-protected implementations of cryptographic primitives.

Constant-time absolute differences. We compute $\left|A_{\ell}-A_{h}\right|$ as follows: First perform a subtraction of $A_{\ell}-A_{h}$ which costs $k$ subtraction instructions. Then we clear a register and perform a subtraction with borrow of zero from this register to obtain a register with the value $t_{A}=0 \mathrm{xff}$ if $A_{\ell}<A_{h}$ or $t_{A}=0$ otherwise. We then xor $t_{A}$ to all $k$ result registers of the subtraction $A_{\ell}-A_{h}$. If $t_{A}=0$, this does not change anything; if $t_{A}=0 \mathrm{xff}$, this produces the ones' complement. We then negate $t_{A}$ (obtaining either $t_{A}=1$ or $t_{A}=0$ ), add it to the lowest of the $k$ registers and ripple the carry through to obtain the two's complement. The whole computation costs $k+1 \mathrm{SUB} / \mathrm{SBC}$ instructions, one CLR instruction, $k$ EOR instructions, one NEG instruction, and $k$ ADD/ADC instructions adding up to a total of $3 k+3$ instructions accounting for $3 k+3$ cycles. The computation of $\left|B_{\ell}-B_{h}\right|$ is done in the same way. We obtain the value of $t$ required for the conditional negation of $M$ as $t=t_{A} \oplus t_{B}$.

Constant-time conditional negation. The most obvious way to compute $L+H-\hat{M}$, given $M$, is to use a conditional branch that either adds or subtracts $M$, depending on the value of $t$. Note that the EOR instruction which we use to compute $t$ sets the zero flag, which we can then use for the branch condition. On many platforms such a conditional branch would inevitably create a timing leak. The AVR does not have any branch-prediction mechanisms; and we can balance the time taken in each of the two branches through NOP instructions to eliminate timing leaks. We implemented this approach and in the following refer to it as the "branched" approach.

There are multiple reasons to avoid branches in cryptographic software. In our port of $\mathrm{NaCl}$ to the AVR archi- 
tecture described in [10] we avoid all secret-data-dependent branches primarily because reviewing NOP-balanced branches for timing leaks is tedious work and argued that avoiding such branches incurs only small penalties. Furthermore, secret-data-dependent branches are often an easy target for safe-error attacks. See, for example, Yen and Joye who described these attacks in [27]. A careful analysis of different multiplication methods from a side-channel point of view is outside the scope of this paper, but we believe that eliminating secret-data-dependent branches is generally a good practice.

An alternative, branch-free way to perform conditional negation is to use the same technique that we used for constant-time absolute differences above (without the initial subtraction). The additions required to convert from the ones' complement to the two's complement can be merged with the additions that are required to combine the partial results; we simply move the bit to the carry flag and replace one ADD instruction by an ADC instruction.

We recommend the branch-free approach for applications that handle secret data and the slightly faster branched approach for applications that do not handle secret data, e.g., signature verification.

Refined Karatsuba multiplication. Combining the partial results in the last step as $L+2^{8 k}(L+H+M)+2^{8 n} H$ looks like $3 n$-byte or $6 k$-byte additions plus rippling a carry bit to the end. However, observe that the byte at position $k$ of the result is obtained as $r_{k}=\ell_{k}+m_{0}+\ell_{0}+h_{0}$; the byte at position $n$ is obtained as $r_{n}=h_{0}+m_{k}+\ell_{k}+h_{k}$. What looks like 6 additions can be reduced to 5 additions by precomputing $s=h_{0}+\ell_{k}$ and then obtaining $r_{k}=m_{0}+\ell_{0}+s$ and $r_{n}=m_{k}+h_{k}+s$. The same trick applies to $r_{k+1}$ and $r_{n+1}$ and so on and saves a total of $k$ additions. We learned this trick from a Crypto 2009 paper by Bernstein [2, Section 2].

An additional advantage of refined Karatsuba is that we can merge the additions of $h_{0}+\ell_{k}, h_{1}+k_{k+1}$ etc. into the multiplication $H=A_{h} \cdot B_{h}$. This is not an advantage from the point of view of purely arithmetic cost, but it simplifies register allocation as explained in Section 4. Note that $H+$ $\left(\ell_{k}, \ldots, \ell_{n-1}\right)$ cannot overflow, the result fits into $n$ bytes.

However, there is also a slight disadvantage of merging this accumulation of $\left(\ell_{k}, \ldots, \ell_{n-1}\right)$. The carry bit that may result from the accumulation is immediately rippled into $h_{k}, \ldots, h_{n-1}$. Later we add $\left(\ell_{0}, \ldots, \ell_{k-1}, h_{k}, \ldots, h_{n-1}\right)$ into the result with an offset of $k$ bytes and subtract $\left(\hat{m}_{0}, \ldots, \hat{m}_{n-1}\right)$ with the same offset. The addition may produce a carry bit $c$ which needs to be rippled to the end; the subtraction may produce a borrow bit $b$ which needs to be rippled to the end. One can also think of this as a carry bit $d=b+c$ which is either 0,1 , or -1 ; The fact that this carry bit can be negative is a direct consequence of merging the addition of $\left(\ell_{k}, \ldots, \ell_{n-1}\right)$ into the multiplication $H=A_{h} \cdot B_{h}$ and rippling the resulting carry. The non-merged computation of $\left(\ell_{0}, \ldots, \ell_{n-1}\right)+\left(h_{0}, \ldots, h_{n-1}\right)-\left(\hat{m}_{0}, \ldots, \hat{m}_{n-1}\right)$ would always produce a non-negative carry, which can simply be rippled to the end.

Merging carries and borrows. If we independently rippled a carry bit $b \in\{0,1\}$ and a borrow bit $c \in\{-1,0\}$ to the end of the result, we would essentially lose the arithmetic benefit of refined Karatsuba. What we do instead is to first compute $b$, then, after subtraction of $\left(\hat{m}_{0}, \ldots, \hat{m}_{n-1}\right)$, use an SBCI of zero from $b$ to obtain $d \in\{-1,0,1\}$ and set the carry bit if and only if $d=-1$. We then clear another register $f$ and perform an SBCI of zero from this register. Now the register pair $(d, f)$ contains $(-1,-1),(0,0)$ or $(1,0)$. Now we can ripple the carry to the end of the result through one addition of $d$ and then subsequent additions with carry of $f$.

Putting it together. The overall arithmetic cost of (branched) Karatsuba multiplication on AVR is thus composed of the following parts:

- One CLR instruction to produce a zero register

- The cost of computing $L$ (multiplication of two $k$-byte integers);

- the cost of computing $M$ (multiplication of two $k$-byte integers);

- the cost of computing $H+\left(\ell_{k}, \ldots, \ell_{n-1}\right)$ (essentially the cost a $k$-byte integer multiplication and $k$ addition instructions);

- $2 k+2$ SUB/SBC instructions, $2 k$ EOR instructions, $2 \mathrm{NEG}$ instructions, and $2 k \mathrm{ADD} / \mathrm{ADC}$ instructions to compute two absolute differences $\left|A_{\ell}-A_{h}\right|$ and $\left|B_{\ell}-B_{h}\right|$;

- $n+1 \mathrm{ADD} / \mathrm{ADC}$ instructions to add $\left(\ell_{0}, \ldots, \ell_{k-1}, h_{k} \ldots, h_{n-1}\right.$ to the result and remember the carry bit;

- one EOR instruction to compute $t$ and set the zero flag if $t=0$;

- one BRNE instruction;

- if the branch is not taken (1 cycle for BRNE):

$-n+2 \mathrm{SUB} / \mathrm{SBC}$ instructions to subtract $M$ and produce carry register pair $(d, f)$;

- one RJMP instruction ( 2 cycles);

- if the branch is taken ( 2 cycles for BRNE):

$-n+1 \mathrm{ADD} / \mathrm{ADC}$ instructions and one CLR instruction to add $M$ and produce carry register pair $(d, f)$;

- one NOP instruction;

- $k$ ADD/ADC instructions to ripple the carry in $(d, f)$ to the end.

In the example of multiplying two 48-bit integers (i.e., $k=3$, see also Appendix A) the computation of $L$ and $M$ costs 40 cycles each (cmp. Table 1 , the cost is slightly lower because we can replace some CLR instructions by MOVW from a zero register pair; this becomes more efficient for multiple multiplications). The computation of $H+\left(\ell_{3}, \ldots, \ell_{5}\right)$ costs 44 cycles. Overall we obtain a cost of 169 cycles from arithmetic (and branch) instructions. This is 18 cycles faster than 
fully unrolled product-scanning multiplication and 3 cycles faster than our optimized 48-bit multiplication (cmp. Table 1). Note that the overhead from loads and stores in this case is the same for all three approaches: 12 loads of input bytes and 12 stores of outputs; 48-bit Karatsuba multiplication does not need any spills as detailed in Section 4. The 3 -cycle gain is small and probably of merely theoretic interest (in particular because Karatsuba multiplication requires more registers), but the gain becomes larger for bigger inputs.

\section{Karatsuba multiplication on the AVR ATmega}

As shown in the previous section, Karatsuba multiplication needs fewer arithmetic instructions than, e.g., fully unrolled product scanning already for very small input sizes. However, it is yet unclear how this arithmetic cost translates to an overall cost including the cost for loads and stores. This section explains our strategies to make efficient use of the available registers and the specifics of the AVR instruction set to keep the overhead from load and store instructions low. Beware that the overall instruction count for multiplication cannot be obtained by adding the lower bound on arithmetic instructions derived in Section 3 to the memory-access overhead explained here. We sometimes spend additional arithmetic instructions to avoid expensive memory access. For example, we occasionally use product-scanning multiplication instead of our optimized small multiprecision routines at the tail of Karatsuba recursion because it uses fewer registers. The complete cycle counts for multiprecision multiplication on AVR are reported in Section 5. All instruction counts in this section refer to the branched variant of our software.

\subsection{One level of Karatsuba}

For the small cases of 1-level Karatsuba multiplication we manage to keep all temporary values in registers; we only need one load instruction per input byte and one store instruction per output byte. The overall strategy we use to achieve this is as follows. First load the $k$ bytes of $A_{\ell}$ and the $k$ bytes of $B_{\ell}$ and compute the $2 k$-byte product $L=A_{\ell} \cdot B_{\ell}$. At this point we have $4 k$ registers occupied. Next, we load the $k$ bytes of $A_{h}$ and the $k$ bytes of $B_{h}$ and overwrite $A_{\ell}$ and $B_{\ell}$ with the absolute differences $\left|A_{\ell}-A_{h}\right|$ and $\left|B_{\ell}-B_{h}\right|$. At this time we have $6 k$ registers occupied. Next, we compute $H+$ $\left(l_{k}, \ldots, l_{n-1}\right)$. This frees the $k$ registers holding $l_{k}, \ldots, l_{n-1}$ and produces the $2 k$-byte output $H$; we thus have $7 k$ live registers at this point. What follows is the computation of $M$, which produces a $2 k$-byte result but also frees the $2 k$ registers containing the absolute differences $\left|A_{\ell}-A_{h}\right|$ and
$\left|B_{\ell}-B_{h}\right|$. Now we have $7 k$ live registers containing all values we need to compute the final result. The only remaining operations are accumulating $\left(l_{0}, \ldots, l_{k-1}, h_{k}, \ldots, h_{n-1}\right)$ and either adding or subtracting $M$.

Remember that 6 of the 32 registers are used for pointers to the two inputs and to the output. Remember further that the register pair $(\mathrm{R} 0, \mathrm{R} 1)$ is used for the output of each MUL instruction. This leaves 24 registers freely available. For the 48-bit multiplication we have $k=3$ and thus $7 k=21$ comfortably fit into these 24 registers (see also Appendix A). For the 64-bit multiplication $(k=4)$ we exploit the fact that immediately after all input bytes are loaded we need only $6 k=24$ registers. Afterwards we can also use the 4 registers holding the pointers to the inputs, i.e., $\mathrm{X}$ and $\mathrm{Y}$, and thus have $7 k=28$ registers available. For the 80 -bit multiplication, we decided to additionally spill one byte of the $\mathrm{Z}$ output address-pointer to (and from) the stack and to use it during the Karatsuba multiplication (which saves 5 clock cycles because it avoids costly moving of intermediate register values). The 96-bit multiplication needs all available registers including all 6 address-pointer bytes (X, Y, and Z) and we spilled two intermediate values in addition to the stack.

For 80-bit and 96-bit Karatsuba multiplication we need more memory operations than strictly necessary to load inputs and store outputs. However, for those input sizes the benefit of smaller arithmetic complexity is so large that Karatsuba still clearly outperforms fully unrolled product scanning. To keep the overhead from memory access small for these variants we avoid spills to the stack as much as possible and rather load inputs (or already completed and stored bytes of output) again. Also, we save a register for the condition bit $t$ by storing it into the T flag. We then use the BRTS branch instruction for conditional addition or subtraction of $M$. For the 96-bit multiplication we use the T flag again to remember the carry bit which comes out of the accumulation of $\left(l_{0}, \ldots, l_{k-1}, h_{k}, \ldots, h_{n-1}\right)$.

In total, the 48-bit multiplication needs the following memory instructions: 12 LD/LDD instructions, and 12 ST/STD instructions. The 64-bit multiplication needs 16 LD/LDD instructions, and 16 ST/STD instructions. The 80-bit multiplication needs 25 LD/LDD instructions, and 20 ST/STD instructions. The 96-bit multiplication needs 42 LD/LDD instructions, 26 ST/STD instructions, 4 PUSH instructions, and 4 POP instructions.

\subsection{Two levels of Karatsuba}

For input sizes of 128, 160, and 192 bits we use two levels of Karatsuba recursion. That means that we use the 1-level Karatsuba multiplication routines described above as building blocks. The general strategy to perform 2-level Karatsuba multiplication is similar to the 1-level Karatsuba multiplications but requires additional spills (PUSH and POP) to 
the stack. First, we calculate $L=A_{\ell} \cdot B_{\ell}$ using 1-level Karatsuba. This requires all available registers. At the end of this multiplication, we store the first $k$ bytes of the result in memory because they are already part of the final result. The remaining $k$ bytes $\ell_{k}, \ldots, \ell_{n-1}$ of $L$ are added on the fly during the calculation of $H=A_{h} \cdot B_{h}$ according to the refined Karatsuba technique described in Section 3. The result is then stored in memory and all registers are available for further processing. After that, we calculate the absolute differences of the input operands, i.e., $\left|A_{\ell}-A_{h}\right|$ and $\left|B_{\ell}-B_{h}\right|$. For 128-bit Karatsuba multiplication, we can keep all $2 k$ bytes of these differences in registers, and perform the third 1level Karatsuba multiplication to obtain $M$. For the 160 and 192-bit Karatsuba multiplication we have to spill some the $2 k$ registers of the absolute differences to the stack to have enough registers available in the computation of $M$. After the computation of $M$, we hold all result bytes in registers and accumulate/subtract the partial products as follows. First, we load $\ell_{0}, \ldots, \ell_{k-1}$ into available registers. For 128-bit Karatsuba, we need $2 k=16$ registers for storing $M$ and $k=8$ for storing $\ell_{0}, \ldots, \ell_{k-1}$. The remaining 6 registers are used for intermediate values during accumulation ( 2 registers are reserved for the result address pointer stored in the $\mathrm{Z}$ register). First, we add or subtract $m_{0}, \ldots, m_{k-1}$ from $\ell_{0}, \ldots, \ell_{k-1}$. Then, we load $h_{0}, \ldots, h_{k-1}$ in chunks of 2 bytes into registers and add them to the intermediate result. The obtained 2-byte results are then stored into memory. Second, we add or subtract $m_{k}, \ldots, m_{n-1}$ to the already loaded values $h_{0}, \ldots, h_{k-1}$. Finally, the registers holding $m_{0}, \ldots, m_{k-1}$ are overwritten with $h_{k}, \ldots, h_{n-1}$. These values are then added to the intermediate result and stored into memory. At the end, the possible carry or borrow is rippled through the already loaded values $h_{k}, \ldots, h_{n-1}$.

Note that we can only directly use the 1-level Karatsuba routines in 2-level Karatsuba because we use subtractive Karatsuba. Also note that on-the-fly accumulation of $\ell_{k}, \ldots, \ell_{n-1}$ into $H$ is not straight forward in 2-level Karatsuba, which computes $H$ itself with 1-level Karatsuba. In the 1-level Karatsuba computation of $H$, we split accumulation of $\ell_{k}, \ldots, \ell_{n-1}$ into two parts: We accumulate $\ell_{k}, \ldots, \ell_{1.5 k-1}$ into $h_{0}, \ldots, h_{k / 2-1}$ and store the result in memory. After that, we accumulate $\ell_{1.5 k}, \ldots, \ell_{n-1}$ again into $h_{0}, \ldots, h_{k / 2-1}$. This accumulation picks up the carry which we obtained in the accumlation of $\ell_{k}, \ldots, \ell_{1.5 k-1}$, adds it into $h_{0}$ and ripples it through $h_{1}, \ldots, h_{k / 2-1}$. The carry from the accumulation of $\ell_{1.5 k}, \ldots, \ell_{n-1}$ is pushed to the stack and later rippled through $H$.

For 160 and 192-bit Karatsuba, the address pointers have to be spilled to and from the stack before/after each 1-level Karatsuba multiplication. Instead of using the PUSH and POP instructions, we load the stack pointer address into either $X$, $\mathrm{Y}$, or $\mathrm{Z}$ using the IN instruction and operate on the stack with LD/LDD and ST/STD instructions. The overhead of this is small and is outweighed by the more flexible addressing of stack space.

In total, the 128-bit multiplication needs 92 LD/LDD instructions, $51 \mathrm{ST} / \mathrm{STD}$ instructions, 2 PUSH instructions, and 2 POP instructions. The 160-bit multiplication needs 144 LD/LDD instructions, 91 ST/STD instructions, 16 PUSH instructions, 18 POP instructions, and 4 IN instructions to copy the stack pointer to Z. The 192-bit multiplication needs 247 LD/LDD instructions, 121 ST/STD instructions, 46 PUSH instructions, 21 POP instructions, 8 IN instructions, and 2 OUT instructions.

\subsection{Three levels of Karatsuba}

We implemented the 256-bit multiplication using 3-levels of Karatsuba. Due to the high register usage of the 1 and 2-level Karatsuba blocks there is almost no room to hold and re-use registers. Thus, we store all result obtained from the 2-level Karatsuba multiplications in the memory and load the values at the end of calculation $M$. Also all absolute differences are pushed to the stack and are loaded during the final 2level Karatsuba multiplication. The obtained results for $M$ are also pushed to the stack and loaded at the end of the multiplication.

In total, the 256-bit multiplication needs the following memory instructions: 352 LD/LDD instructions, 204 ST/STD instructions, 82 PUSH instructions, 114 POP instructions, 8 IN instructions, and 32 OUT instructions.

\section{Results}

This section reports cycle counts, code size, and stack usage for the software presented in this paper. All cycle counts are obtained through simulation in the Atmel AVR Studio version 5.0.1223. All multiplication routines passed tests on 1000 random inputs and passed a test with all input bytes set to 255. These tests were performed on an ATmega2560 (Arduino MEGA development board).

Like previous papers we report cycle counts, code sizes, and stack usage excluding the function-call cost, i.e., the cost for CALL, RET, initial PUSH and final POP of caller registers, 3 MOVW instructions required to copy the function arguments to the X, Y, Z registers, and the cost to clear register $\mathrm{R} 1$ before returning from the function.

It is important to note that for small input sizes, product scanning does not use all available registers and can avoid some of the PUSHs and POPs of caller registers. A function that only multiplies, e.g., two 48-bit integers and follows the $\mathrm{C}$ function-call ABI for AVR will thus be faster when using product scanning than our Karatsuba multiplication. However, the 48-bit Karatsuba multiplication will be faster if it is used in a larger (inlined) context. See also Section 4. 
Table 2 Speed and size comparison of multiprecision multiplication on AVR ATmega. All counts exclude function-call overhead.

\begin{tabular}{|c|c|c|c|c|c|c|c|c|c|}
\hline & & & & & Input & ize (bits & & & \\
\hline Approach & & 48 & 64 & 80 & 96 & 128 & 160 & 192 & 256 \\
\hline Unrolled product scanning: & cycles & 235 & 395 & 595 & 836 & - & - & - & - \\
\hline & bytes & 350 & 598 & 910 & 1288 & - & - & - & - \\
\hline Operand caching [11]: & cycles & - & - & - & - & - & $2393^{a}$ & $3467^{a}$ & $6121^{a}$ \\
\hline & bytes & - & - & - & - & - & $3696^{a}$ & $5354^{a}$ & $9476^{a}$ \\
\hline Consecutive operand caching [22]: & cycles & - & - & - & - & 1532 & 2356 & 3464 & 6180 \\
\hline & bytes & - & - & - & - & N/A & 3652 & N/A & N/A \\
\hline (Consecutive) operand caching [23]: & cycles & - & - & - & - & - & 2346 & 3437 & 6115 \\
\hline & bytes & - & - & - & - & - & 3632 & N/A & N/A \\
\hline This paper (branched): & cycles & $217^{b}$ & $360^{b}$ & $522^{b}$ & $780^{b}$ & $1325^{c}$ & $1998^{c}$ & $2923^{c}$ & $4797^{d}$ \\
\hline & bytes & $348^{b}$ & $580^{b}$ & $828^{b}$ & $1228^{b}$ & $2228^{c}$ & $3262^{c}$ & $4602^{c}$ & $8022^{d}$ \\
\hline & stack & 0 & 0 & 0 & 4 & 1 & 19 & 36 & 58 \\
\hline This paper (branch-free): & cycles & $222^{b}$ & $368^{b}$ & $533^{b}$ & $800^{b}$ & $1369^{c}$ & $2054^{c}$ & $2987^{c}$ & $4961^{d}$ \\
\hline & bytes & $342^{b}$ & $576^{b}$ & $826^{b}$ & $1226^{b}$ & $2156^{c}$ & $3150^{c}$ & $4492^{c}$ & $7614^{d}$ \\
\hline & stack & 0 & 0 & 0 & 4 & 1 & 19 & 36 & 58 \\
\hline${ }^{a}$ Counts obtained using the & ne & & & & & & & & \\
\hline${ }^{b}$ One level of Karatsuba & & & & & & & & & \\
\hline${ }^{c}$ Two levels of Karatsuba & & & & & & & & & \\
\hline Three levels of Karatsuba & & & & & & & & & \\
\hline
\end{tabular}

A summary of our results, together with the best previous results from the literature, is presented in Table 2. All implementations listed in this table focus on speed and are fully unrolled. For input sizes from 48 to 96 bits we are not aware of any results from the literature achieving better speeds than fully unrolled product-scanning multiplication. For those input sizes we include a comparison with fully unrolled product scanning. For 48-bit inputs this is not optimal as demonstrated by our optimized multiplication routine (see Section 3 and Appendix B). We believe that also for 64-bit, 80-bit, and 96-bit inputs, careful optimization of quadratic-complexity multiplication can gain a few cycles compared to fully unrolled product scanning. However, we do not expect those gains to be larger than what we gain by using subquadratic-complexity Karatsuba multiplication.

Hutter and Wenger make the software described in [11] available through an online code generator at http: //www . iaik.tugraz.at/content/research/vlsi/projects/ mulopcache/. The cycle counts of the software generated by this online tool are slightly lower than the ones reported in the paper. We compare to the improved cycle counts.

\section{Conclusion and future work}

In this paper we presented new speed records for multiplication of integers from 48 bits up to 256 bits on AVR ATmega. We showed that carefully optimized Karatsuba multiplication technique is more efficient than quadratic-complexity multiplication already for much smaller input sizes than previously believed.

The most obvious future work is to apply the multiplication routines described in this paper to elliptic-curve cryptography. For example, in [18], Liu, Seo, Großschädl, and Kim use consecutive operand-caching multiplication to push the performance boundaries for arithmetic on the NIST-P192 curve It will be straight-forward to push the boundaries even further by replacing consecutive operand-caching multiplication by our Karatsuba multiplication routines.

Furthermore, this paper focuses on speed of multiplication routines without considering the size of the implementation. It will be interesting to investigate tradeoffs between speed and size for Karatsuba multiplication on AVR, for example by implementing the small multiprecision multiplications at the bottom of the recursion only once and use jumps or calls to this routine. Another direction of future research is to examine whether the Karatsuba technique can also speed up squaring on AVR. Finally, we hope that the techniques described in this paper will serve as an inspiration to re-examine possible performance gains from Karatsuba multiplication for relatively small inputs on other embedded platforms.

\section{References}

1. Paul Barrett. Implementing the Rivest Shamir and Adleman public key encryption algorithm on a standard digital signal processor. In Andrew M. Odlyzko, editor, Advances in Cryptology - 
CRYPTO '86, volume 263 of Lecture Notes in Computer Science, pages 311-323. Springer-Verlag Berlin Heidelberg, 1987. 2

2. Daniel J. Bernstein. Batch binary Edwards. In Shai Halevi, editor, Advances in Cryptology - CRYPTO 2009, volume 5677 of Lecture Notes in Computer Science, pages 317-336. Springer-Verlag Berlin Heidelberg, 2009. http://cr.yp.to/papers.html\#bbe. 5

3. Paul G. Comba. Exponentiation cryptosystems on the IBM PC. IBM Systems Journal, 29(4), 1990. http://lyle.smu.edu/ seidel/courses/cse8351/papers/CombaCRYPTO.pdf. 2

4. Martin Fürer. Faster integer multiplication. SIAM Journal on Computing, 39(3):979-1005, 2009. 1

5. Conrado P. L. Gouvêa and Julio López. Software implementation of pairing-based cryptography on sensor networks using the MSP430 microcontroller. In Nicolas Sendrier Bimal Roy, editor, Progress in Cryptology - INDOCRYPT 2009, volume 5922 of Lecture Notes in Computer Science, pages 248-262. Springer-Verlag Berlin Heidelberg, 2009. http://conradoplg.cryptoland. net/files/2010/12/indocrypt09.pdf. 2

6. Conrado P. L. Gouvêa, Leonardo B. Oliveira, and Julio López. Efficient software implementation of public-key cryptography on sensor networks using the MSP430X microcontroller. Journal of Cryptographic Engineering, 2(1), 2012. http://conradoplg. cryptoland.net/files/2010/12/jcen12.pdf. 2

7. Johann Großschädl, Roberto M. Avanzi, Erkay Savaş, and Stefan Tillich. Energy-efficient software implementation of long integer modular arithmetic. In Josyula R. Rao and Berk Sunar, editors, Cryptographic Hardware and Embedded Systems - CHES 2005, volume 3659 of Lecture Notes in Computer Science, pages 75-90. Springer-Verlag Berlin Heidelberg, 2005. www. iacr.org/ archive/ches2005/006.pdf. 2

8. Nils Gura, Arun Patel, Arvinderpal Wander, Hans Eberle, and Sheueling Chang Shantz. Comparing elliptic curve cryptography and RSA on 8-bit CPUs. In Marc Joye, editor, Cryptographic Hardware and Embedded Systems - CHES 2004, volume 3156 of Lecture Notes in Computer Science, pages 119132. Springer-Verlag Berlin Heidelberg, 2004. www.iacr.org/ archive/ches2004/31560117/31560117.pdf. 2

9. Nils Gura and Lawrence A. Spracklen. Hybrid multi-precision multiplication. United States Patent 7650374, 2010. Application filed Nov. 23, 2004, http://www.freepatentsonline.com/ 7650374.html. 2

10. Michael Hutter and Peter Schwabe. $\mathrm{NaCl}$ on 8-bit AVR microcontrollers. In Amr Youssef and Abderrahmane Nitaj, editors, Progress in Cryptology - AFRICACRYPT 2013, volume 7918 of Lecture Notes in Computer Science, pages 156-172. Springer-Verlag Berlin Heidelberg, 2013. http://cryptojedi. org/papers/\#avrnacl. 2, 5

11. Michael Hutter and Erich Wenger. Fast multi-precision multiplication for public-key cryptography on embedded microprocessors. In Bart Preneel and Tsuyoshi Takagi, editors, Cryptographic Hardware and Embedded Systems - CHES 2011, volume 6917 of Lecture Notes in Computer Science, pages 459-474. SpringerVerlag Berlin Heidelberg, 2011. https://online.tugraz.at/ tug_online/voe_main2. getvolltext?pCurrPk=58138. 2, 8

12. Michael Hutter and Erich Wenger. Multiplication of large operands. WIPO Patent Application WO/2013/044276, 2013. Application filed Sep.27, 2011, http://www.freepatentsonline. com/W02013044276A1. html. 2

13. Anatolii Karatsuba and Yuri Ofman. Multiplication of multidigit numbers on automata. Soviet Physics Doklady, 7:595-596, 1963. Translated from Doklady Akademii Nauk SSSR, Vol. 145, No. 2, pp. 293-294, July 1962. Scanned version on http://cr.yp.to/ bib/1963/karatsuba.html. 1

14. Anatolii A. Karatsuba. The complexity of computations. Proceedings of the Steklov Institute of Mathematics, 211:169-183, 1995. http://www.ccas.ru/personal/karatsuba/divcen.pdf. 1
15. Christian Lederer, Roland Mader, Manuel Koschuch, Johann Großschädl, Alexander Szekely, and Stefan Tillich. Energy-efficient implementation of ecdh key exchange for wireless sensor networks. In Olivier Markowitch, Angelos Bilas, Jaap-Henk Hoepman, Chris J. Mitchell, and Jean-Jacques Quisquater, editors, Information Security Theory and Practice, volume 5746 of Lecture Notes in Computer Science, pages 112-127. Springer-Verlag Berlin Heidelberg, 2009. http://www.cs.bris.ac.uk/ tillich/pdf/ Lederer2009Energy-EfficientImplementation.pdf. 3

16. Zhe Liu and Johann Großschädl. New speed records for Montgomery modular multiplication on 8-bit AVR microcontrollers. Cryptology ePrint Archive, Report 2013/882, 2013. https:// eprint. iacr. org/2013/882/. 3

17. Zhe Liu, Johann Großschädl, and Ilya Kizhvatov. Efficient and side-channel resistant RSA implementation for 8-bit AVR microcontrollers. In Proceedings of the 1st International Workshop on the Security of the Internet of Things - SECIoT'10, 2010. https://www.nics.uma.es/seciot10/files/ pdf/liu_seciot10_paper.pdf. 2, 3

18. Zhe Liu, Hwajeong Seo, Johann Großschädl, and Howon Kim. Efficient implementation of NIST-compliant elliptic curve cryptography for sensor nodes. In Sihan Qing, Jianying Zhou, and Dongmei Liu, editors, Information and Communications Security, volume 8233 of Lecture Notes in Computer Science, pages 302317. Springer-Verlag Berlin Heidelberg, 2013. http://orbilu. uni.lu/bitstream/10993/12934/1/ICICS2013.pdf. 8

19. Krishnaji S. Patwardhan, Somashekara A. Naimpally, and Shyam L. Singh. Lülāvtī of Bhāskarācārya. Motilal Banarsidass Publishers, 2001. http://books.google.com/books? id=AoX5q7JjM2kC. 2

20. Arnold Schönhage and Volker Strassen. Schnelle Multiplikation großer Zahlen. Computing, 7(3):281-292, 1971. 1

21. Michael Scott and Piotr Szczechowiak. Optimizing multiprecision multiplication for public key cryptography. Cryptology ePrint Archive, Report 2007/299, 2007. https://eprint.iacr.org/ 2007/299/. 2

22. Hwajeong Seo and Howon Kim. Multi-precision multiplication for public-key cryptography on embedded microprocessors. In Dong Hoon Lee MotiYung, editor, Information Security Applications, volume 7690 of Lecture Notes in Computer Science, pages 55-67. Springer-Verlag Berlin Heidelberg, 2012. http://isaa.sch.ac.kr/wisa2012/\%EB\%85\%BC\%EB\%AC\%B8/ Session\%202/1-130_Multi-precision\%20Multiplication $\% 20$ for $\% 20$ Public-Key $\% 20$ Cryptography $\% 20$ on $\% 20$ Embedded $\% 20$ Microprocessors. pdf. 2, 8

23. Hwajeong Seo and Howon Kim. Optimized multi-precision multiplication for public-key cryptography on embedded microprocessors. International Journal of Computer and Communication Engineering, 2(3), 2013. http://www.ijcce.org/papers/ 183-J034.pdf. 2, 8

24. Laurence E. Sigler. Fibonacci's Liber Abaci-Leonardo Pisano's Book of Calculation. Springer-Verlag New York, 2003. http:// books. google. com/books? id=PilhoG JeKBUC. 2

25. Frank J. Swetz. Capitalism and Arithmetic: The New Math of the 15th Century. Open Court, 1987. 2

26. Andrei L. Toom. The complexity of a scheme of functional elements realizing the multiplication of integers. Soviet Mathematics Doklady, 3:714-716, 1963. www.de.ufpe.br/ ${ }^{\sim}$ toom/ my-articles/engmat/MULT-E.PDF. 1

27. Sung-Ming Yen and Marc Joye. Checking Before Output May Not Be Enough Against Fault-Based Cryptanalysis. In IEEE Transactions on Computers, volume 49 of IEEE Transactions on Computers, pages 967-970. IEEE Computer Society, 2000. 5 
A Karatsuba multiplication of two 48-bit numbers

Listing 1 Karatsuba multiplication of 48-bit integer at address X and 48-bit integer at address Y; 96-bit result is written to address Z

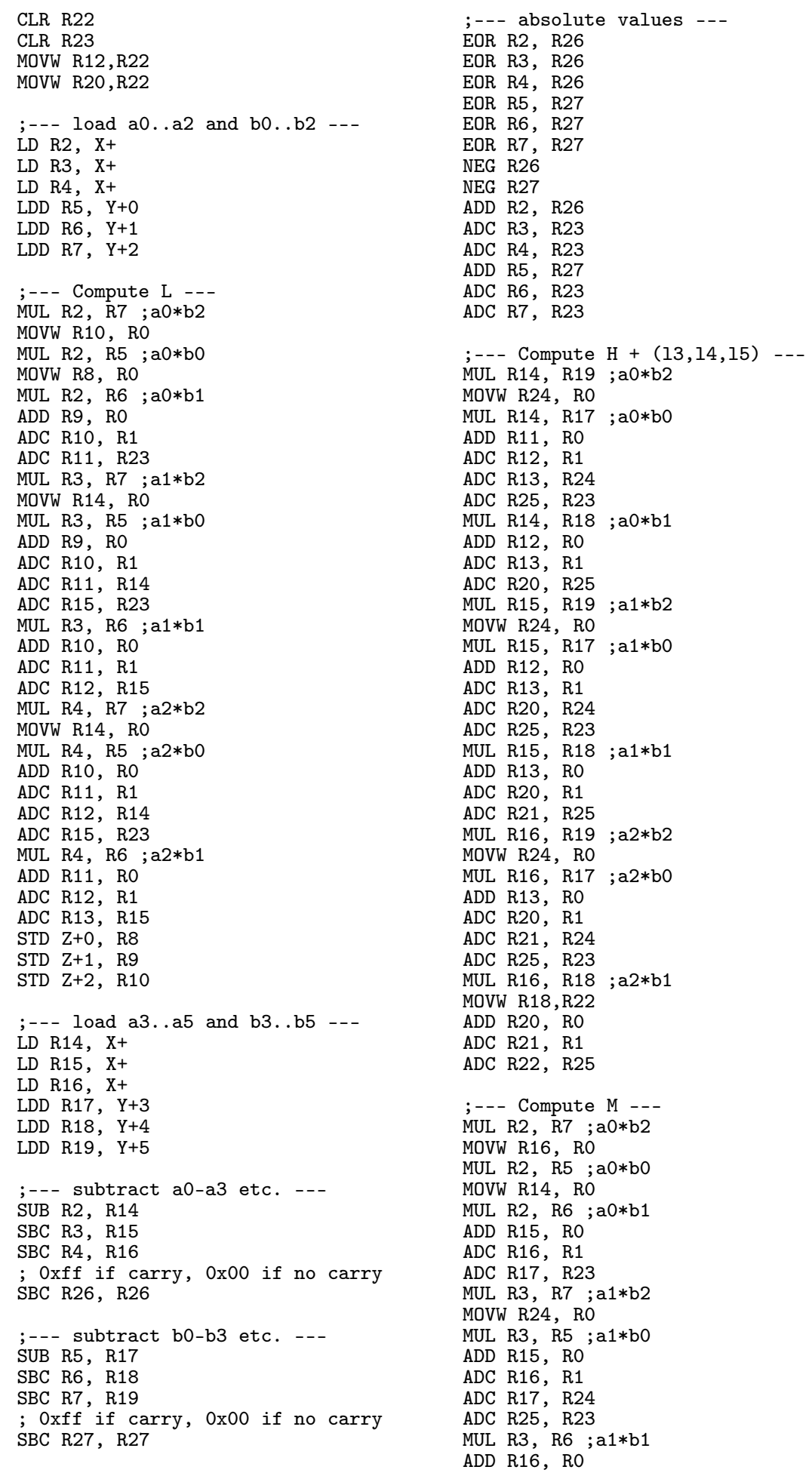

ADC R17, R1

ADC R18, R25

MUL R4, R7 ; a $2 * \mathrm{~b} 2$

MOVW R24, R0

MUL R4, R5 ; a $2 * b 0$

ADD R16, R0

ADC R17, R1

ADC R18, R24

ADC R25, R23

MUL R4, R6 ; a2*b1

ADD R17, R0

ADC R18, R1

ADC R19, R25

;- - add $13+$ h0 to h3 etc. -.

ADD R8, R11

ADC R9, R12

ADC R10, R13

ADC R11, R20

ADC R12, R21

ADC R13, R22

; store carry in R23

ADC R23, R23

;--- load sign bit ---

EOR R26, R27

BRNE add_M

; subtract $M$

SUB R8, R14

SBC R9, R15

SBC R10, R16

SBC R11, R17

SBC R12, R18

SBC R13, R19

SBCI R23, 0

SBC R24, R24

;R23:R24 is $-1,0$, or 1

RJMP final

add_M :

ADD R8, R14

ADC R9, R15

ADC R10, R16

ADC R11, R17

ADC R12, R18

ADC R13, R19

CLR R24

ADC R23, R24

NOP ; constant time

final:

STD $Z+3, R 8$

STD Z+4, R9

STD Z+5, R10

STD Z+6, R11

STD $Z+7$, R12

STD Z+8, R13

;--- ripple carry ---

ADD R20, R23

ADC R21, R24

ADC R22, R24

STD Z+9, R20

STD Z+10, R21

STD Z+11, R22

ADD R16, R0 
B Small multiprecision multiplications

Listing 2 Optimized multiplication of two 24-bit integers, input $A$ in registers R2,R3,R4; input $B$ in registers R7,R8,R9; result in registers R12,R13,R14,R15,R16,R17.

\begin{tabular}{lll}
\hline CLR R23 & MUL R3, R9 & LD R2, X+ \\
& MOVW R24, R0 & MUL R4, R9 \\
MUL R2, R9 & MOVW R24, R0 \\
MOVW R14, R0 & MUL R3, R7 & \\
& ADD R13, R0 & MUL R4, R7 \\
MUL R2, R7 & ADC R14, R1 & ADD R14, R0 \\
MOVW R12, R0 & ADC R15, R24 & ADC R15, R1 \\
MUL R2, R8 & ADC R25, R23 & ADC R16, R24 \\
ADD R13, R0 & MUL R3, R8 & ADC R25, R23 \\
ADC R14, R1 & ADD R14, R0 & MUL R4, R8 \\
ADC R15, R23 & ADC R15, R1 & ADD R15, R0 \\
& ADC R16, R25 & ADC R16, R1 \\
CLR R16 & & ADC R17, R25 \\
LD R2, X+ & CLR R17 & \\
& & \\
\hline
\end{tabular}

Listing 3 Optimized multiplication of two 32bit integers, input $A$ in registers $R 2, R 3, R 4, R 5$; input $B$ in registers $\mathrm{R} 7, \mathrm{R} 8, \mathrm{R} 9, \mathrm{R} 10$; result in registers R12,R13,R14,R15,R16,R17,R18,R19.

\begin{tabular}{|c|c|c|}
\hline CLR R23 & MUL R3, R8 & ADD R17, R25 \\
\hline & ADD R14, R0 & ADC R18, R0 \\
\hline MUL R2, R9 & ADC R15, R1 & ADC R19, R1 \\
\hline MOVW R14, R0 & ADC R25, R23 & \\
\hline MUL R2, R7 & MUL R4,R10 & MUL R5, R8 \\
\hline MOVW R12, RO & $\begin{array}{l}\text { ADD R16, R25 } \\
\text { ADC R17, R0 }\end{array}$ & MOVW R24, RO \\
\hline MUL R2, R8 & ADC R18, R1 & MUL $R 4, R 8$ \\
\hline ADD R13, R0 & & ADD R15, R0 \\
\hline ADC R14,R1 & & ADC R24, R1 \\
\hline ADC R15, R23 & $\begin{array}{ll}\text { CLR } & \text { R19 } \\
\text { MUL } & \text { R4, R9 }\end{array}$ & ADC R25, R23 \\
\hline $\begin{array}{l}\text { MUL R3, R10 } \\
\text { MOVW R16, R0 }\end{array}$ & MOVW R24, RO & $\begin{array}{l}\text { MUL R5,R7 } \\
\text { ADD R15, R0 }\end{array}$ \\
\hline & MUL $\mathrm{R} 4, \mathrm{R} 7$ & ADC R24, R1 \\
\hline & ADD R14, R0 & ADC R25, R23 \\
\hline CLR R18 & ADC R15, R1 & \\
\hline MUL R2, R10 & ADC R16, R24 & MUL R5, R9 \\
\hline MOVW R24, RO & ADC R25, R23 & $\begin{array}{l}\text { ADD R16, R24 } \\
\text { ADC R0, R25 }\end{array}$ \\
\hline MUL R3, R7 & MUL R3, R9 & ADC R1, R23 \\
\hline ADD R13, R0 & ADD R15, R0 & ADD R17, R0 \\
\hline ADC R14, R1 & ADC R16, R1 & ADC R18, R1 \\
\hline ADC R15, R24 & ADC R25, R23 & ADC R19, R23 \\
\hline ADC R25, R23 & MUL R5, R10 & \\
\hline
\end{tabular}

Listing 4 Optimized multiplication of two 40-bit integers, input $A$ in registers $\mathrm{R} 2, \mathrm{R} 3, \mathrm{R} 4, \mathrm{R} 5, \mathrm{R} 6$; input $B$ in registers $\mathrm{R} 7, \mathrm{R} 8, \mathrm{R} 9, \mathrm{R} 10, \mathrm{R} 11$; result in registers R12,R13,R14,R15,R16,R17,R18,R19,R20,R21.

\begin{tabular}{|c|c|c|}
\hline $\begin{array}{ll}\text { CLR } & \text { R18 } \\
\text { CLR } & \text { R19 }\end{array}$ & ADC R18, R1 & MUL R5, R8 \\
\hline MOVW R20, R18 & MUL R3, R10 & $\mathrm{ADD} R 16, \mathrm{R} 0$ \\
\hline & ADD R16, R0 & ADC R17, R1 \\
\hline MUL R2, R9 & ADC R17, R1 & ADC R25, R21 \\
\hline MOVW R14, R0 & ADC R18, R21 & MUL R5, R11 \\
\hline MUL R2, R7 & & ADD R18, R25 \\
\hline MOVW R12, R0 & $\begin{array}{l}\text { MUL R4, R9 } \\
\text { MOVW R24, R0 }\end{array}$ & $\begin{array}{l}\text { ADC R19, R0 } \\
\text { ADC R20, R1 }\end{array}$ \\
\hline MUL R2, R8 & & \\
\hline ADD R13, R0 & MUL R4, R7 & MUL R5, R10 \\
\hline ADC R14, R1 & ADD R14, R0 & ADD R18, R0 \\
\hline ADC R15, R21 & $\begin{array}{lll}\text { ADC } & \text { R15, } & \text { R1 } \\
\text { ADC } & \text { R16, } & \text { R24 }\end{array}$ & $\begin{array}{lll}\text { ADC } & \text { R19, } & \text { R1 } \\
\text { ADC } & \text { R20, } & \text { R21 }\end{array}$ \\
\hline MUL R2, R11 & ADC R25, R21 & \\
\hline MOVW R16, R0 & & $\begin{array}{l}\text { MUL R6, R9 } \\
\text { MOVW R24, R0 }\end{array}$ \\
\hline MUL R2, R10 & ADD R15, R0 & \\
\hline ADD R15, R0 & ADC R16, R1 & MUL R6, R7 \\
\hline ADC R16, R1 & ADC R25, R21 & ADD R16, R0 \\
\hline ADC R17, R21 & & ADC R17, R1 \\
\hline & MUL R4, R11 & ADC R18, R24 \\
\hline & ADD R17, R25 & ADC R25, R21 \\
\hline MUL R3, R9 & ADC R18, R0 & \\
\hline MOVW R24, R0 & ADC R19, R1 & $\begin{array}{ll}\text { MUL } & R 6, \mathrm{R} 8 \\
\text { ADD } & \mathrm{R} 17, \mathrm{R} 0\end{array}$ \\
\hline MUL R3, R7 & MUL R4, R10 & ADC R18, R1 \\
\hline ADD R13, R0 & ADD R17, R0 & ADC R25, R21 \\
\hline ADC R14, R1 & ADC R18, R1 & \\
\hline ADC R15, R24 & ADC R19, R21 & MUL R6, R10 \\
\hline ADC R25, R21 & & 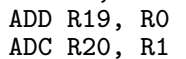 \\
\hline $\begin{array}{l}\text { MUL } R 3, \text { R8 } \\
\text { ADD } R 14, \quad \text { R0 }\end{array}$ & $\begin{array}{l}\text { MUL R5, R9 } \\
\text { MOVW R24, R0 }\end{array}$ & ADC R21, R21 \\
\hline ADC R15, R1 & & MUL R6, R11 \\
\hline ADC R25, R21 & $\begin{array}{l}\text { MUL R5, R7 } \\
\text { ADD R15, R0 }\end{array}$ & 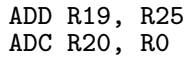 \\
\hline MUL R3, R11 & ADC R16, R1 & $\mathrm{ADC} R 21, \mathrm{R} 1$ \\
\hline ADD R16, R25 & ADC R17, R24 & \\
\hline ADC R17, R0 & ADC R25, R21 & \\
\hline
\end{tabular}


Listing 5 Optimized multiplication of two 48-bit integers, input $A$ in registers $\mathrm{R} 2, \mathrm{R} 3, \mathrm{R} 4, \mathrm{R} 5, \mathrm{R} 6, \mathrm{R} 7$; input $B$ in registers $R 8, R 9, R 10, R 11, R 12, R 13$; result in registers R14,R15,R16,R17,R18,R19,R20,R21,R22,R23,R24,R25.

\begin{tabular}{|c|c|c|}
\hline CLR R20 & MUL R4,R10 & MUL R6,R10 \\
\hline CLR R21 & MOVW R26, RO & MOVW R26, R0 \\
\hline MOVW R22, R20 & & \\
\hline MOVW R24, R20 & $\begin{array}{ll}\text { MUL } & R 4, R 8 \\
\text { ADD } & \text { R } 16, R 0\end{array}$ & $\begin{array}{l}\text { MUL } R 6, R 8 \\
\text { ADD } 18, R 0\end{array}$ \\
\hline MUL R2,R10 & ADC R17,R1 & ADC R19,R1 \\
\hline \multirow[t]{2}{*}{ MOVW R16, R0 } & ADC R18,R26 & ADC R20,R26 \\
\hline & ADC R27,R25 & ADC R27, R25 \\
\hline \multicolumn{3}{|l|}{ MUL R2, R8 } \\
\hline \multirow[t]{2}{*}{ MOVW R14, R0 } & MUL R4,R9 & MUL R6,R9 \\
\hline & ADD R17, R0 & ADD R19, R0 \\
\hline MUL R2,R9 & ADC R18,R1 & ADC R20,R1 \\
\hline ADD R15, R0 & ADC R27, R25 & ADC R27, R25 \\
\hline \multicolumn{3}{|l|}{ ADC R16, R1 } \\
\hline \multirow[t]{2}{*}{ ADC R17, R25 } & MUL R4,R12 & MUL R6,R12 \\
\hline & ADD R19, R27 & ADD R21, R27 \\
\hline MUL R2,R12 & ADC R20,R0 & ADC R22, R0 \\
\hline \multirow[t]{2}{*}{ MOVW R18, R0 } & ADC R21, R1 & ADC R23, R1 \\
\hline & ADC R22,R25 & ADC R24, R25 \\
\hline \multicolumn{3}{|l|}{ MUL R2, R11 } \\
\hline ADD R17,R0 & MUL R4,R11 & MUL R6,R11 \\
\hline ADC R18,R1 & MOVW R26, R0 & MOVW R26, R0 \\
\hline ADC R19, R2b & MUL R4,R13 & MUL R6,R13 \\
\hline MUL R2, R13 & ADD R19, R26 & ADD R21, R26 \\
\hline ADD R19, R0 & ADC R20,R27 & $\mathrm{ADC} \quad \mathrm{R} 22, \mathrm{R} 27$ \\
\hline \multirow{2}{*}{ ADC R20, R1 } & $\mathrm{ADC} R 21, \mathrm{R} 0$ & $\mathrm{ADC} R 23, \mathrm{R} 0$ \\
\hline & $\mathrm{ADC} R 22, \mathrm{R} 1$ & ADC R24,R1 \\
\hline \multicolumn{3}{|l|}{ MUL R3, R10 } \\
\hline MOVW R26, R0 & MUL R5, R10 & MUL R7, R10 \\
\hline & MOVW R26, RO & MOVW R26, R0 \\
\hline \multicolumn{3}{|l|}{ MUL R3, R8 } \\
\hline ADD R15, R0 & MUL R5, R8 & MUL R7,R8 \\
\hline ADC R16,R1 & ADD R17, R0 & ADD R19,R0 \\
\hline ADC R17, R26 & ADC R18,R1 & ADC R20,R1 \\
\hline \multirow[t]{2}{*}{ ADC R27, R25 } & ADC R19,R26 & ADC R21, R26 \\
\hline & ADC R27, R25 & ADC R27, R25 \\
\hline \multicolumn{3}{|l|}{ MUL R3, R9 } \\
\hline ADD R16, R0 & MUL R5, R9 & MUL R7, R9 \\
\hline ADC R17, R1 & ADD R18, R0 & ADD R20, RO \\
\hline \multirow[t]{2}{*}{ ADC R27, R25 } & ADC R19, R1 & ADC R21, R1 \\
\hline & ADC R27, R25 & ADC R27, R25 \\
\hline \multicolumn{3}{|l|}{ MUL R3,R12 } \\
\hline ADD R18,R27 & MUL R5,R12 & MUL R7,R12 \\
\hline ADC R19, R0 & ADD R20, R27 & ADD R22, R27 \\
\hline ADC R20,R1 & ADC R21, R0 & $\mathrm{ADC}$ R23, R0 \\
\hline \multirow[t]{2}{*}{ ADC R21, R25 } & ADC R22, R1 & $\mathrm{ADC} R 24, \mathrm{R} 1$ \\
\hline & ADC R23, R25 & ADC R25, R25 \\
\hline \multicolumn{3}{|l|}{ MUL R3,R11 } \\
\hline \multirow{2}{*}{ MOVW R26, RO } & MUL R5,R11 & MUL R7, R11 \\
\hline & MOVW R26, R0 & MOVW R26, R0 \\
\hline \multicolumn{3}{|l|}{ MUL R3,R13 } \\
\hline ADD R18, R26 & MUL $R 5, R 13$ & MUL $R 7, R 13$ \\
\hline ADC R19, R27 & ADD R20,R26 & ADD R22, R26 \\
\hline ADC R20,R0 & ADC R21,R27 & ADC R23, R27 \\
\hline ADC R21,R1 & ADC R22,R0 & ADC R24, R0 \\
\hline & ADC R23,R1 & ADC R25, R1 \\
\hline
\end{tabular}

